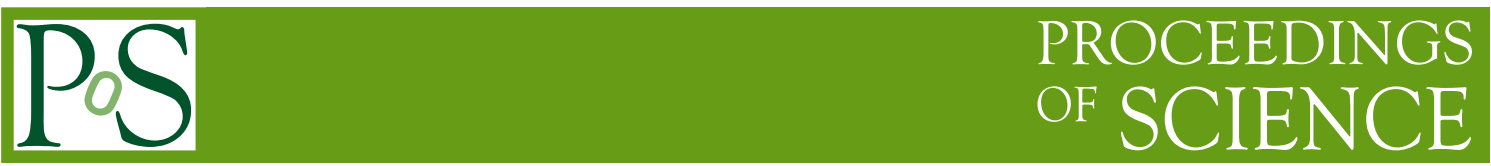

\title{
Use and misuse of ChPT in the heavy-light systems *
}

\author{
Damir Bećirević ${ }^{\dagger}$ \\ Laboratoire de Physique Théorique (Bât. 210) \\ Université Paris-Sud, Centre d'Orsay \\ F-91405 Orsay-Cedex, France \\ E-mail: Damir.Becireviceth.u-psud.fr
}

\section{Svjetlana Fajfer}

J. Stefan Institute, Jamova 39, P.O.Box 3000, and

Department of Physics, University of Ljubljana,

Jadranska 19, 1000 Ljubljana, Slovenia

E-mail: Svjetlana.Fajfer@ijs.si

\section{Jernej Kamenik}

INFN, Laboratori Nazionali di Frascati

I-00044 Frascati, Italy

E-mail: Jernej.Kamenik@lnf.infn.it

We discuss the range of validity of chiral perturbation theory when applied to the systems of heavy-light mesons. Having in mind the recent experimental evidence according to which the heavy-light scalar and axial states are closer to the ground states than anticipated, we revisited the prediction for the chiral behavior of the $B_{q}^{0}-\bar{B}_{q}^{0}$ mixing amplitude and examined the impact of nearness of the $(1 / 2)^{+}$states. We conclude that the standard ChPT expressions with $N_{F}=3$ light flavours are not useful in guiding the extrapolation of hadronic quantities computed on the lattice. Instead those derived in HMChPT with $N_{F}=2$, i.e., including only the pion loops, are still adequate as long as they are applied to the pions lighter than $350 \mathrm{MeV}$, or the quarks lighter than a third of the physical strange quark mass.

The XXV International Symposium on Lattice Field Theory

July 30-4 August 2007

Regensburg, Germany

*Supported in part by the EU Contract No. MRTN-CT-2006-035482, "Flavianet".

† Speaker. 


\section{Introduction}

In this note we summarise the findings of our research presented in ref. [1]. The fact that the lattice QCD community is heavily dependent on the formulae derived in chiral perturbation theory (ChPT) to extrapolate the directly accessible results for almost any phenomenologically relevant quantity to the physical -nearly chiral- limit, requires a clear assessment of the validity of ChPT. Only then one can be able to claim "a high precision physical result" deduced from the lattice QCD simulations combined with ChPT. Before touching on the peculiarities related to the heavy-light mesons, we will make a short trip to the sector of light mesons because the first warnings of how far we should (should not) push ChPT in terms of precision requirements already show there.

\subsection{Light mesons}

The viability of chiral expansion in the theory with $N_{F}=3$ light flavours $(u, d$, and $s)$ has been questionable since the very beginning of the theory. The main reason is that the strange quark mass is half-a-way between the chiral limit and $\Lambda_{\mathrm{QCD}}$, which might significantly lower the ChPT order parameters $\left[\lim _{m_{u, d, s} \rightarrow 0}\left(f_{\pi},\langle\bar{q} q\rangle\right)\right]$, with respect to their $N_{F}=2$ counterparts, and thus spoil the perturbative nature of the chiral expansion (a number of situations in which the next to leading order term in the chiral expansion is larger than the leading one). Of course, the issue can be settled after confronting the experimental data to the ChPT formulae, but the quality of the actual $K \pi$ scattering data is still not good enough to resolve this issue (a special worry is related to the low energy constants $L_{4}$ and $L_{6}$ ). As of now, it is safe to say that the formulae derived in ChPT with $N_{F}=3$ are not sufficiently reliable to seek $\mathscr{O}(1 \%)$ precision when extrapolating the lattice data to the physical limit.

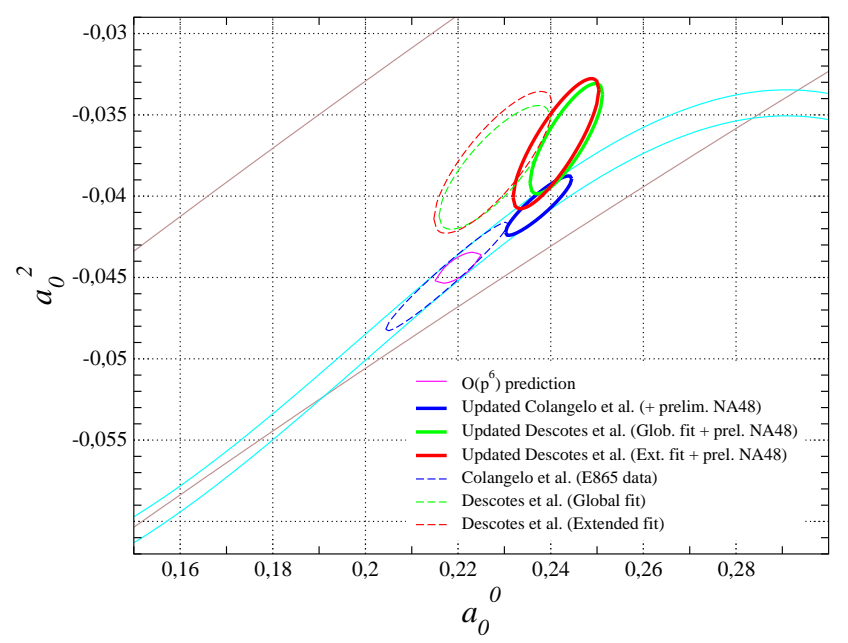

Figure 1: $S$-wave scattering lengths $a_{0}^{I}$ (isospin $I=0,2$ ). The dashed (thick) ellipses are obtained by using the old (new) experimental $K_{e 4}$-data. The two sets of ellipses refer to two different analyses [2, 3] while the small one, in the centre of the plot, is the 2-loop ChPT prediction (Courtesy of S.Descotes-Genon).

The situation with $N_{F}=2$ is different simply because that theory has been tested. Back in 2001, such a test looked like a triumph: the experimentally measured $S$-wave scattering lengths 
of $\pi \pi$-system, emerging from $K_{e 4}$ decay, were fully consistent with the NNLO ChPT formula. It was deduced that the the Gell-Mann-Oakes-Renner formula (GMOR), $m_{\pi}^{2}=2 B_{0} m_{u, d}+\ldots$, is saturated by the leading term to more than $94 \%$ [2]. However, that situation changed since the new and improved $K_{e 4}$ data, collected in NA48, appeared. If one repeats exactly the same analysis as that of ref. [2] with those new data then "only" about $85 \%$ of the GMOR formula is saturated by the first term, which can be read off from the plot shown in fig. 1.

In spite of the speculations that the electromagnetic and isospin breaking corrections might patch up the new conclusion we believe it is fair to summarise that ChPT with pions only $\left(N_{F}=2\right)$ passed the experimental tests but the level of accuracy is still controversial. Instead, the actual situation in the $N_{F}=3$ case remains unclear.

\subsection{Heavy-light mesons}

ChPT has been extensively applied to describe the dynamics of light constituents in the heavylight mesons. In particular, heavy-meson ChPT (HMChPT) has been constructed in the static heavy quark limit [4], with only one extra parameter, $g$, the coupling of a pseudo-Goldstone boson (PGB) to a doublet of lowest lying heavy-light mesons $\left[j_{\ell}^{P}=(1 / 2)^{-}\right]$, namely the pseudoscalar $\left(J^{P}=0^{-}\right)$and vector $\left(1^{-}\right)$mesons. HMChPT with $N_{F}=2$ and $N_{F}=3$ light flavours inherit the problems discussed above. An extra complication appeared after the experimenters reported on the observation of the orbitally excited states $\left[j_{\ell}^{P}=(1 / 2)^{+}\right]$, both scalar $\left(0^{+}\right)$and axial $\left(1^{+}\right)$ones. The observations are made in the case of mesons with the charmed heavy quark [5], to which the confirmation came from the unquenched lattice study in the static heavy quark limit [6]. In summary the splitting between the excited and ground states is only

$$
\Delta_{S_{s}} \equiv m_{D_{0 s}^{*}}-m_{D_{s}}=m_{D_{1 s}}-m_{D_{s}^{*}}=350 \mathrm{MeV}, \quad \Delta_{S_{q}} \approx 430(30) \mathrm{MeV}
$$

for the strange and the non-strange case respectively. Leaving aside the reasons why $\Delta_{S_{s}} \neq \Delta_{S_{q}}$, it is clear that $\Delta_{S_{s, q}}<\Lambda_{\chi}, m_{\eta}, m_{K}$, and thus the basic assumption that no resonances appear between zero and $\Lambda_{\chi}$ is simply not correct in HMChPT. ${ }^{1}$

In view of importance of the HMChPT guidance to extrapolating the lattice results to reach the phenomenologically relevant physical quantities, such as $f_{B}$, or $B^{0}-\bar{B}^{0}$ mixing parameters, we revised the derivation of these expressions in HMChPT and studied the impact of $\Delta_{S}$ on the chiral behavior of the $B_{d}^{0}-\bar{B}_{d}^{0}$ mixing amplitude. The special attention is given to $B_{d}^{0}-\bar{B}_{d}^{0}$, rather than $B_{s}^{0}-\bar{B}_{s}^{0}$, because of its better potential for the new physics search [8].

\section{Computation of $f_{B} \sqrt{m_{B}} \rightarrow \hat{f}_{q}$}

In HMChPT, in the static heavy quark limit $\left(m_{Q} \rightarrow \infty\right)$, the pseudoscalar decay constant is of dimension $3 / 2$, and schematically we write $\lim _{m_{B} \rightarrow \infty} f_{B_{q}} \sqrt{m_{B_{q}}} \rightarrow \hat{f}_{q}$. Its light quark dependence is expected to be described by the chiral loops shown in fig. 2 , and the result reads

$$
\hat{f}_{d}=\hat{f}_{0}\left[1-\frac{1+3 g^{2}}{4(4 \pi f)^{2}}\left(3 m_{\pi}^{2} \log \frac{m_{\pi}^{2}}{\mu^{2}}+2 m_{K}^{2} \log \frac{m_{K}^{2}}{\mu^{2}}+\frac{1}{3} m_{\eta}^{2} \log \frac{m_{\eta}^{2}}{\mu^{2}}\right)+\text { c.t. }\right],
$$

\footnotetext{
${ }^{1}$ The fact that $\Delta_{S_{s}}<\Delta_{S_{q}}$ is still craving for an explanation. It cannot be reconciled with results from the lattice simulations, nor with the HMChPT considerations [7].
} 
where $\hat{f}_{0}$ is the heavy-light meson decay constant in the chiral limit, $g$ is the coupling of the heavylight mesons doublet to a PGB (also in the chiral limit) are the new parameters are the two constants coming from the weak current and from the lagrangian. "c.t." stands for the local counterterms the $\mu$-dependence of which cancels against the one present in the chiral logarithms.

A tacit assumption in deriving the above formula is that there is a clear separation by which the chiral logarithms describe the long distance dynamics whereas the local counterterms encode the information on short distance physics. The lattice results obtained at larger $m_{q}>m_{d}$ are expected to provide a vi-
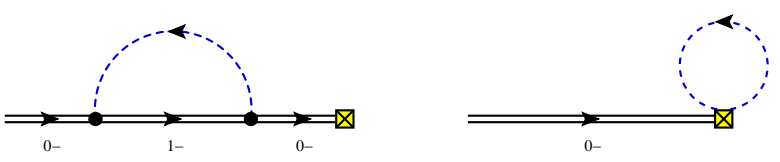

Figure 2: The chiral loop corrections to $\hat{f}_{d}$ : the box denotes the weak current vertex, double line is the heavylight meson and the dashed line is the PGB propagator. able method to fix those counterterms, i.e., to compute the associated low energy constants. An equivalent statement is that HMChPT can be used to guide the extrapolation of $\hat{f}_{q}$ computed for $m_{q}>m_{d}$ to the physical limit.
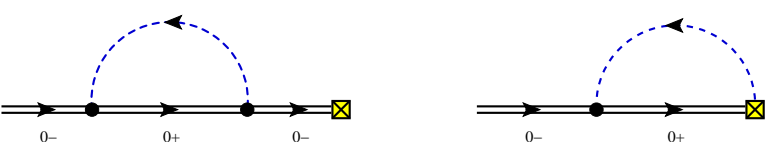

Figure 3: Inclusion of the scalars in the chiral corrections to $\hat{f}_{d}$. Notice that $m_{0^{+}}-m_{0^{-}}=\Delta_{S}<\Lambda_{\chi}$.
Specifying the separation scale means distinguishing the particles which can propagate in the chiral loops from those which cannot. In eq. (2.1) that scale is evidently assumed to be $\Lambda_{\chi} \gtrsim m_{\eta} \cdot{ }^{2}$ But the fact that $\Delta_{S_{s, q}} \lesssim m_{K, \eta}$, and thus also $\Delta_{S_{s, q}}<\Lambda_{\chi}$, means that one must include the propagation of the

scalars in the loops as well. The lagrangian and the weak current that include the effect of $(1 / 2)^{+}$ states are given in ref. [1]. The only two new diagrams that contribute to $\hat{f}_{d}$ are those shown in fig. 3 and there are only two new couplings that appear in the new -extended to include scalarexpression,

$$
\begin{aligned}
\hat{f}_{q}= & \hat{f}_{0}\left\{1+\sum_{i} \frac{t_{q a}^{i} a_{a q}^{i \dagger}}{2(4 \pi f)^{2}}\left[3 g^{2} \lim _{x \rightarrow 0} \frac{d}{d x}\left[x J_{1}\left(m_{i}^{2}, x\right)\right]-I_{1}\left(m_{i}^{2}\right)-h^{2}\left(J_{1}\left(m_{i}^{2}, \Delta_{S}\right)+J_{2}\left(m_{i}^{2}, \Delta_{S}\right)\right.\right.\right. \\
& \left.\left.\left.+\Delta_{S} \frac{d}{d \Delta_{S}}\left(J_{1}\left(m_{i}^{2}, \Delta_{S}\right)+J_{2}\left(m_{i}^{2}, \Delta_{S}\right)\right)\right)-2 h \frac{\hat{f}_{0}^{+}}{\hat{f}_{0}}\left(I_{1}\left(m_{i}^{2}\right)+I_{2}\left(m_{i}^{2}, \Delta_{S}\right)\right)\right]+ \text { c.t. }\right\},
\end{aligned}
$$

are $h$, the coupling of the PGB to one $(1 / 2)^{-}$and one $(1 / 2)^{+}$heavy-light meson, and $\hat{f}_{0}^{+}$, the weak decay constant of the orbitally excited heavy-light meson in the chiral limit. If one takes the limit $m_{i}<\Delta_{S}$, which only applies to pions, there is an amusing automatic separation between the $\Delta_{S}$-dependent terms from the independent ones. The result is that the HMChPT with the pion loops "survive" whereas the $K$ - and $\eta$-loops are drowned in a whole lot of new terms of which it is significant the presence of those proportional to $\Delta_{S}^{2} \log \left(\Delta_{S}^{2} / \mu^{2}\right)$, thus competitive in size with the $K$ - and $\eta$-contributions. This definitely restricts the applicability of the HMChPT formulae to the light quark masses corresponding to pions lighter than $\Delta_{S}$. That can be converted to a condition that the light quark masses to which the HMChPT formulae apply should be lighter than a third

\footnotetext{
${ }^{2}$ The propagation of $1^{-}$heavy-light state does not give any contribution because we take it to be degenerate in mass with the pseudoscalar meson, which holds true in the static heavy quark limit.
} 
of the physical strange quark mass, i.e., $m_{q}<m_{s}^{\text {phys. }} / 3$, and only to such data the HMChPT with $N_{F}=2$ flavours can be used, or

$$
\hat{f}_{q}=\hat{f}_{0}\left[1-\frac{1+3 g^{2}}{2(4 \pi f)^{2}} \frac{3}{2} m_{\pi}^{2} \log \frac{m_{\pi}^{2}}{\mu^{2}}+c_{f}(\mu) m_{\pi}^{2}\right]
$$

with $c_{f}(\mu)$ being the combination of low energy constants that multiply $m_{\pi}^{2}$ and which, together with $g$ and $\hat{f}_{0}$, should be fixed by fitting the lattice data to this expression. Otherwise, i.e., if we do not restrict to $m_{q}<m_{s}^{\text {phys. }} / 3$, the number of parameters in the expression rises from 3 to 13 .

\section{Bag Parameters}

The Standard Model (SM) bag parameter in the static heavy quark limit is defined via $\left\langle\bar{B}_{q}^{0}|\widetilde{O}(v)| B_{q}^{0}\right\rangle=$ $(8 / 3) \hat{f}_{q}(v)^{2} \widetilde{B}_{q}(v)$, where the $\Delta F=2$ operator $\widetilde{O}=\left(\bar{b}_{v} \gamma_{\mu}^{L} q\right)\left(\bar{b}_{v} \gamma_{\mu}^{L} q\right) \equiv \gamma_{\mu}^{L} \otimes \gamma_{L}^{\mu}$. Assuming its UVphysics ( $v$-scale dependence) is being taken care of, say in HQET perturbation theory, one can study its bosonised form in HMChPT,

$$
\widetilde{O}=\sum_{X} \beta_{1 X} \operatorname{Tr}\left[\left(\xi \bar{H}^{Q}\right)_{q} \gamma_{\mu}\left(1-\gamma_{5}\right) X\right] \times \operatorname{Tr}\left[\left(\xi H^{\bar{Q}}\right)_{q} \gamma^{\mu}\left(1-\gamma_{5}\right) X\right]+\text { ct. },
$$

where $X \in\left\{1, \gamma_{5}, \gamma_{v}, \gamma_{v} \gamma_{5}, \sigma_{v \rho}\right\}$, and the field $H(v)$ describes the $(1 / 2)^{-}$-doublet, consisting of the pseudoscalar and vector heavy-light mesons. Once the scalar/axial fields are introduced in HMChPT the expressions become much more complicated and essentially useless for a meaningful numerical study. In ref. [1] we showed that for $m_{q}<m_{s}^{\text {phys. }} / 3$, we again can separate the contribution of the pion loop corrections and lump everything else into the finite counterterms. Before giving the explicit expressions, let us show on a specific example how that separation occurs. ${ }^{3}$ Consider a typical (dimensionally regularised) integral that appears in these calculations and expand around the decoupling limit of the positive parity states:

$$
\begin{aligned}
& -2(4 \pi)^{2} v_{\mu} v_{v} \times i \mu^{\varepsilon} \int \frac{d^{4-\varepsilon} p}{(2 \pi)^{4-\varepsilon}} \frac{p^{\mu} p^{v}}{\left(p^{2}-m_{\pi}^{2}\right)\left[\Delta_{S}^{2}-(v p)^{2}\right]} \\
& =-\frac{2\left(4 \pi^{2}\right)}{\Delta_{S}^{2}} v_{\mu} v_{v}\left[i \mu^{\varepsilon} \int \frac{d^{4-\varepsilon} p}{(2 \pi)^{4-\varepsilon}} \frac{p^{\mu} p^{v}}{p^{2}-m_{\pi}^{2}}+\mathscr{O}\left(1 / \Delta_{S}^{2}\right)\right] \longrightarrow-\frac{m_{\pi}^{4}}{2 \Delta_{S}^{2}} \log \frac{m_{\pi}^{2}}{\mu^{2}}+\ldots
\end{aligned}
$$

where the dots stand for terms of higher order in $m_{\pi}^{2} / \Delta_{S}^{2}$. This expansion separates the $N_{F}=2$ chiral loops from the rest, diagram by diagram, as demonstrated in ref. [1]. At the end we arrive at the useful formulas

$$
\begin{aligned}
\widetilde{B}_{q} \hat{f}_{q}^{2} & =\widetilde{B}_{0} \hat{f}_{0}^{2}\left[1-\frac{3 g^{2}+2}{(4 \pi f)^{2}} m_{\pi}^{2} \log \frac{m_{\pi}^{2}}{\mu^{2}}+c_{\mathscr{O}_{1}}(\mu) m_{\pi}^{2}\right] \\
\widetilde{B}_{q} & =\widetilde{B}_{0}\left[1-\frac{1-3 g^{2}}{2(4 \pi f)^{2}} m_{\pi}^{2} \log \frac{m_{\pi}^{2}}{\mu^{2}}+c_{B}(\mu) m_{\pi}^{2}\right] .
\end{aligned}
$$

Note that there is one new counterterm coefficient, $c_{B}(\mu)$, and $\widetilde{B}_{0}=\lim _{m_{q} \rightarrow 0} \widetilde{B}_{q}$, both of which should be fixed from lattice data collected with the light quark $m_{q}<m_{s}^{\text {phys. }} / 3$.

\footnotetext{
${ }^{3}$ This practice is pretty standard in soft collinear effective theory.
} 


\section{Supersymmetric $B^{0}-\bar{B}^{0}$ mixing amplitude}

In SUSY not only $W$-boson propagates in the loop, and thus not only the left-left operator survives at low energies. In the static heavy quark limit there are in fact 4 operators, the matrix elements of which can contribute to the $B^{0}-\bar{B}^{0}$ mixing amplitude. In HMChPT that number further reduces to 3 because the two operators differ only by the gluon exchange which cannot alter the chiral logarithms [9]. The remaining operators are $\widetilde{O}_{2,4}=\mathbb{1}_{L} \otimes \mathbb{1}_{L, R}$, where $\mathbb{1}_{L / R}=\bar{b}_{v}\left(1 \mp \gamma_{5}\right) q$. The bosonised forms of these operators were first correctly figured out in ref. [10], which we then confirmed by deriving them in a somewhat different way [1]. The resulting expressions for the bag parameters, defined as $\left\langle\bar{B}_{q}^{0}\left|\widetilde{O}_{2,4}\right| B_{q}^{0}\right\rangle=\left(b_{2,4} / 3\right) \hat{f}_{q}^{2} \widetilde{B}_{2,4}$, with $b_{2}=-5, b_{4}=7$, are of course different from the SM bag-parameter. In the theory with $N_{F}=2$ light quarks $(u, d)$ we have

$$
\begin{aligned}
\widetilde{B}_{2,4 q} \hat{f}_{q}^{2} & =\widetilde{B}_{2,4}^{\text {Tree }} \hat{f}_{0}^{2}\left[1-\frac{3 g^{2}(3-Y)+3 \pm 1}{2(4 \pi f)^{2}} m_{\pi}^{2} \log \frac{m_{\pi}^{2}}{\mu^{2}}+c_{\mathscr{O}_{2,4}}(\mu) m_{\pi}^{2}\right], \\
\Rightarrow \widetilde{B}_{2,4 q} & =\widetilde{B}_{2,4}^{\text {Tree }}\left[1+\frac{3 g^{2} Y \mp 1}{2(4 \pi f)^{2}} m_{\pi}^{2} \log \frac{m_{\pi}^{2}}{\mu^{2}}+c_{B_{2,4}}(\mu) m_{\pi}^{2}\right],
\end{aligned}
$$

which structurally differs from eq. (3.2) in that the coefficient multiplying the logarithmic contribution here involves a yet another low-energy constant, $Y$, and which is also to be extracted from the fit with the lattice data. In our paper [1] we went through the same steps as above: we presented the expressions obtained in the theory with $N_{F}=3$, then included the effects of the scalars in the loops and showed that a decoupling of the pion piece with respect to the kaon, eta and the contribution of excited heavy-light mesons occurs in this case too. Therefore the useful formulas are those written in eq. (4.1).

\section{Conclusion}

ChPT is nowadays accepted as an effective theory of QCD at very low energies. However, it is a theory solely based on the spontaneous chiral symmetry breaking pattern, $S U\left(N_{F}\right)_{L} \otimes S U\left(N_{F}\right)_{R} \rightarrow$ $S U\left(N_{F}\right)_{V}$, and tells us nothing about confinement. Its elementary objects are PGB's, and not quarks and gluons like in QCD. Therefore an appropriate matching of ChPT to QCD amounts to solving the confinement problem in QCD, as well as that of the spontaneous chiral symmetry breaking at the more fundamental level. In other words, the matching between ChPT and QCD -as of now- is unclear. This is to be contrasted to the case of heavy quark effective theory (HQET) where such a matching at any given order in the $1 / m_{Q}$-expansion can be made and is in fact systematically improvable, order-by-order in $\alpha_{S}\left(m_{Q}\right)$.

Both HQET (expansion in $1 / m_{Q}^{n}$ ) and ChPT (expansion in $p_{\pi}^{2 n}$ ) share the same worry, i.e., how good is their convergence in realistic situations in which one retains only one or two subleading terms in the expansion. ${ }^{4}$ In ChPT with $N_{F}=3$ that issue is still a subject of controversies, while in the $N_{F}=2$ case the experimental tests have been made and the results are quite encouraging although it is still unclear how to interpret this test in terms of accuracy.

From the point of view of lattice QCD practitioners, the chiral behavior predicted by an effective theory for the quantities that are of high phenomenological interest, such as $f_{B}, B^{0}-\bar{B}^{0}$ mixing

\footnotetext{
${ }^{4}$ It is perhaps worth mentioning that unlike in ChPT, where expansion is in $p_{\pi}^{2 n}$, in HMChPT it goes like $p_{\pi}^{n}$.
} 
amplitude, or the $B \rightarrow \pi$ form factors, is a very important guideline when extrapolating the results collected at unphysical light quark masses down to the physical $d / u$-quark mass. The hope is that a result of such an extrapolation has smaller systematic errors.

Spurred by the recent experimental evidence indicating that the heavy-light excited states belonging to the $(1 / 2)^{+}$doublet are much lighter than expected, we revisited the predictions based on the use of HMChPT with $N_{F}=3$ light flavours and in the static heavy quark limit. We showed that in practical applications only the formulas involving the pion loops, i.e. the theory with $N_{F}=2$, should be used. Otherwise the number of low energy constants to be fixed from the lattice data becomes prohibitively large and the contributions due to the presence of the near heavy-light excitations are comparable in size to the ones that are due to kaon- and/or $\eta$-loops. We showed on explicit examples how this decoupling occurs and provided the expressions for $f_{B}$, the standard and SUSY $B^{0}-\bar{B}^{0}$ mixing amplitude, as well as for the couplings $g, h$ [11], the Isgur-Wise function [12], and the scalar meson decay constant [1].

\section{References}

[1] D. Becirevic, S. Fajfer and J. Kamenik, "Chiral behavior of the $B_{s, d}^{0}-\bar{B}_{s, d}^{0}$ mixing amplitude in the standard model and beyond," JHEP 0706 (2007) 003 [hep-ph/ 0612224$]$.

[2] G. Colangelo, J. Gasser and H. Leutwyler, "The quark condensate from $K_{e 4}$ decays," Phys. Rev. Lett. 86 (2001) $5008[$ hep-ph/0103063].

[3] S. Descotes-Genon, N. H. Fuchs, L. Girlanda and J. Stern, "Analysis and interpretation of new low-energy $\pi \pi$ scattering data," Eur. Phys. J. C 24 (2002) 469 [hep-ph/0112088], see also this volume arXiv:0709.0265.

[4] R. Casalbuoni et al., "Phenomenology Of Heavy Meson Chiral Lagrangians," Phys. Rept. 281 (1997) 145 [hep-ph/9605342].

[5] B. Aubert et al. [BABAR], Phys. Rev. Lett. 90 (2003) 242001 [hep-ex/ 0304021 ] ; D. Besson et al. [CLEO], Phys. Rev. D 68 (2003) 032002 [ hep-ex/ 0305100 ] ; K. Abe et al.,[BELLE] Phys. Rev. Lett. 92 (2004) 012002 [hep-ex/ 0307052 ] and Phys. Rev. D 69 (2004) 112002 [hep-ex/ 0307021 ] ; J. M. Link et al. [FOCUS], Phys. Lett. B 586 (2004) 11.

[6] A. M. Green et al. [UKQCD], “Excited B mesons from the lattice,” Phys. Rev. D 69 (2004) 094505 [hep-lat/0312007].

[7] D. Becirevic, S. Fajfer and S. Prelovsek, "On the mass differences between the scalar and pseudoscalar heavy-light mesons,” Phys. Lett. B 599 (2004) 55 [hep-ph/ 0406296 ].

[8] M. Bona et al. [UTfit Collaboration], "Model-independent constraints on Delta $F=2$ operators and the scale of New Physics,", arXiv:0707.0636 [hep-ph] .

[9] D. Becirevic and G. Villadoro, "Remarks on the hadronic matrix elements relevant to the SUSY $K^{0}-\bar{K}^{0}$ mixing amplitude," Phys. Rev. D 70, 094036 (2004) [hep-lat/ 0408029 ] .

[10] W. Detmold and C. J. Lin, "Matrix elements of the complete set of $\Delta B=2$ and $\Delta C=2$ operators in heavy meson chiral perturbation theory,” Phys. Rev. D 76 (2007) 014501 [hep-lat/ 0612028 ]

[11] S. Fajfer and J. Kamenik, "Chiral loop corrections to strong decays of positive and negative parity charmed mesons," Phys. Rev. D 74 (2006) 074023 [hep-ph / 0606278 ] .

[12] J. O. Eeg, S. Fajfer and J. Kamenik, "Chiral loop corrections to weak decays of B mesons to positive and negative parity charmed mesons,” JHEP 0707 (2007) 078 [0705.4567 [hep-ph]]. 